\section{Presentation Type:}

Oral Presentation

Subject Category: MDR GNR

How Does Antimicrobial Resistance Increase Medical Costs in Community-Acquired Acute Pyelonephritis?

Bongyoung Kim; Taul Cheong and Jungmo Ahn

Background: The proportion of antimicrobial-resistant Enterobacterales that are causative pathogens for community-acquired acute pyelonephritis (CAAPN) has been increasing. We examined the effect of antimicrobial resistance on medical costs in CA-APN. Methods: A single-center retrospective cohort study was conducted at a tertiary-care hospital in Korea between January 2018 to December 2019. All hospitalized patients aged $\geq 19$ years who were diagnosed with CA-APN were recruited, and those with Enterobacterales as a causative pathogen were included. Comparisons between CA-APN caused by extended-spectrum $\beta$-lactamase (ESBL)-producing pathogens (ESBL+ group) and those by non-ESBL-producing organisms (ESBL- group) as well as CAAPN caused by ciprofloxacin-resistant pathogens (CIP-R group) and those by
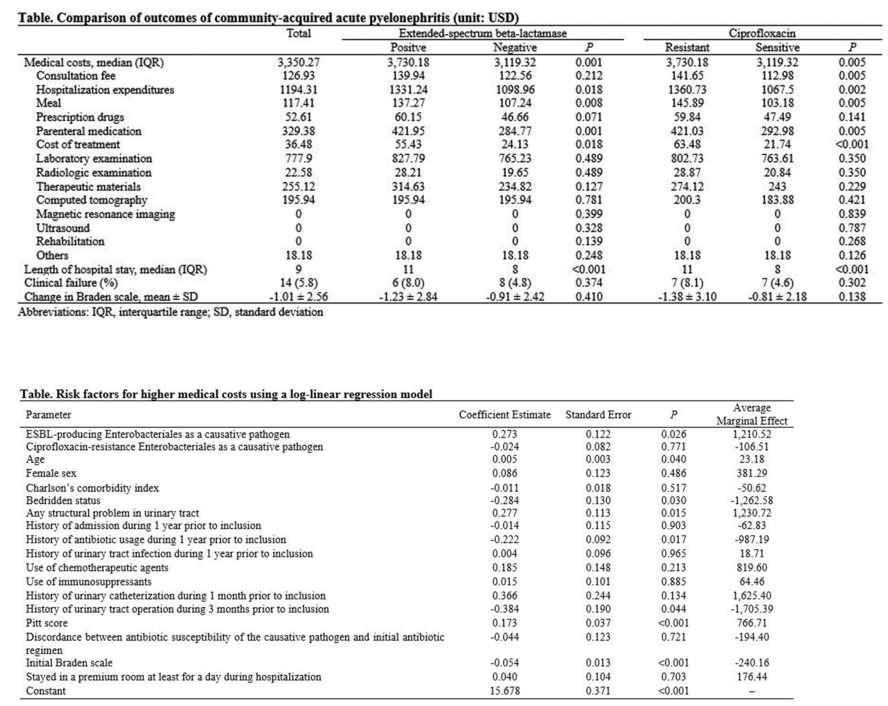
Abbreviations: ESBL, extended-spectrum-beta-lactamase

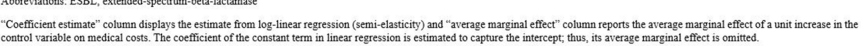

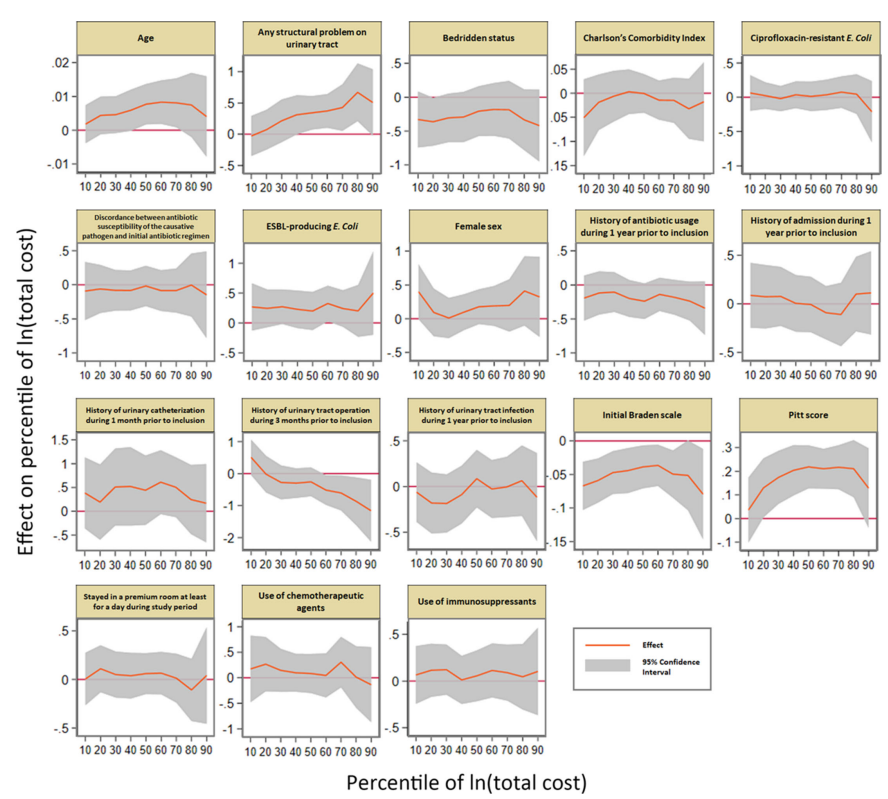

ciprofloxacin-sensitive pathogens (CIP-S group) were performed. Log-linear regression was performed to determine the risk factors for medical costs. Results: In total, 241 patients were included in this study. Of these, 75 (31.1\%) had an ESBL-producing pathogen and 87 (36.1\%) had a ciprofloxacin-resistant pathogen. The overall medical costs were significantly higher in the ESBL+ group compared with the ESBL- group (US $\$ 3,730.18$ vs US $\$ 3,119.32) \mathrm{P}<0.001)$ as well as in CIP-R group compared with CIP-S group $(3,730.18$ USD vs. 3,119.32 USD, $\mathrm{P}=0.005)$. In addition, length of stay was longer in ESBL+ group compared with ESBL-group (11 vs. 8 days, $\mathrm{P}<0.001)$ as well as in CIP-R group compared with CIP-S group (11 vs. 8 days, $\mathrm{P}<0.001)$. There were no significant difference in the proportion of clinical failure between ESBL+ and ESBL- groups; CIP-R and CIP-S groups. Based on the log-linear regression model, the costs associated with ESBL-producing Enterobacterales as the causative pathogen would be, on average, $27 \%$ higher or US $\$ 1,211$ higher than its counterpart $(P=.026)$. By the same token, a patient who is a year older would incur US $\$ 23$ higher cost $(P=.040)$. Having any structural problem in urinary tract would incur US $\$ 1,231$ higher cost $(P=.015)$. A unit increase in Pitt score would incur US\$767 USD higher cost $(\mathrm{P}<0.001)$ higher cost, all other things constant. Conclusions: Medical costs for hospitalized patients with CA-APN are increased by the existence of ESBL-producing Enterobacterales but not by the existence of ciprofloxacin-resistant Enterobacterales.

Funding: No

Disclosures: None

Antimicrobial Stewardship \& Healthcare Epidemiology 2021;1(Suppl. S1):s23

doi:10.1017/ash.2021.42

Presentation Type:

Oral Presentation

Subject Category: Medical Informatics

Automated Nationwide Benchmarking Dashboard for Antimicrobial Stewardship Programs within the Veterans' Health Administration

Michihiko Goto; Eli Perencevich; Alexandre Marra; Bruce Alexander; Brice Beck; Daniel Livorsi; Julia Friberg; Christopher Richards; DeShauna Jones and Michael Sauder

Group Name: VHA Center for Antimicrobial Stewardship and Prevention of Antimicrobial Resistance (CASPAR) Background: Antimicrobial stewardship programs (ASPs) are advised to measure antimicrobial consumption as a metric for audit and feedback. However, most ASPs lack the tools necessary for appropriate risk adjustment and standardized data collection, which are critical for peer-program benchmarking. We created a system that automatically extracts antimicrobial use data and patient-level factors for risk-adjustment and a dashboard to present risk-adjusted benchmarking metrics for ASP within the Veterans' Health Administration (VHA). Methods: We built a system to extract patient-level data for antimicrobial use, procedures, demographics, and comorbidities for acute inpatient and long-term care units at all VHA hospitals utilizing the VHA's Corporate Data Warehouse (CDW). We built baseline negative binomial regression models to perform risk-adjustments based on patient- and unit-level factors using records dated between October 2016 and September 2018. These models were then leveraged both retrospectively and prospectively to calculate observed-to-expected ratios of antimicrobial use for each hospital and for specific units within each hospital. Data transformation and applications of risk-adjustment models were automatically performed within the CDW database server, followed by monthly scheduled data transfer from the CDW to the Microsoft Power BI server for interactive data visualization. Frontline antimicrobial stewards at 10 VHA hospitals participated in the project as pilot users. Results: Separate baseline risk-adjustment models to predict days of therapy (DOT) for all antibacterial agents were created for acute-care and longterm care units based on 15,941,972 patient days and 3,011,788 DOT between October 2016 and September 2018 at 134 VHA hospitals. Risk adjustment models include month, unit types (eg, intensive care unit [ICU] vs non-ICU for acute care), specialty, age, gender, comorbidities (50 and 30 factors for acute care and long-term care, respectively), and preceding procedures ( 45 and 24 procedures for acute care and long-term care, respectively). We created additional models for each antimicrobial category based on National Healthcare Safety Network definitions. For each hospital, risk-adjusted benchmarking metrics and a monthly ranking within the VHA system were visualized and presented to end users

\title{
Figure 1.
}

(c) The Author(s), 2021. Published by Cambridge University Press on behalf of The Society for Healthcare Epidemiology of America. This is an Open Access article, distributed under the terms of the Creative Commons Attribution licence (http://creativecommons.org/licenses/by/4.0/), which permits unrestricted re-use, distribution, and reproduction in any medium, provided the original work is properly cited. 